\title{
Models of Accretion Disks around Young Stars
}

\author{
Paola D'Alessio \\ Centro de Radioastronomía y Astrofísica, Universidad Nacional \\ Autónoma de México, Ap. Postal 3-72, Morelia, Michoacan, México. \\ Nuria Calvet, Lee Hartmann \\ Smithsonian Astrophysical Observatory, 60 Garden Street, MS 42, \\ Cambridge, MA 02138 \\ James Muzerolle \\ Steward Observatory, University of Arizona, 933 N. Cherry Ave., \\ Tucson, AZ 85721 \\ Michael Sitko \\ Department of Physics, University of Cincinnati, Cincinnati, $\mathrm{OH}$ \\ 45221-0011
}

\begin{abstract}
We discuss the importance of accretion in calculating disk models for young stellar objects. In particular, we show that a disk inner rim, irradiated by both the star and the accretion shocks at the stellar surface, can naturally explain recent observations of DG Tau with the Keck interferometer. We present models for two objects, with mass accretion rates differing by almost two orders of magnitude, to illustrate the effects of accretion on the overall disk structure and emission.
\end{abstract}

\section{Introduction}

Disks around Classical T Tauri Stars (CTTS) are accretion disks. This means that a fraction of disk material loses energy and angular momentum, falling into the gravitational potential well of the star. The standard view of the final accretion process in a low mass star is that a well organized stellar magnetic field truncates and couples to the inner disk at a few stellar radii (Königl 1991; Shu et al. 1994). Disk material falls onto the star along the stellar magnetic field lines in a magnetospheric flow, releasing most of the accretion luminosity, $L_{\text {shock }} \sim G M_{*} \dot{M} / R_{*}\left(1-R_{*} / R_{i}\right) \sim L_{a c c}$, at the stellar surface in hot accretion shocks(Calvet \& Gullbring 1998, CG98). The emission of these shocks produce a blue/UV continuum excess that veils the stellar photospheric absorption lines. Detailed measurement of veiling excesses, combined with models of the hot continuum, have resulted in a long list of mass accretion rate estimates in CTTS (see Calvet, Hartmann \& Strom 2000, and references therein). In addition, models of the gas infalling through the magnetosphere have successfully reproduced blue 
shifted emission peaks, blue line asymmetries and red shifted absorption components of emission lines in CTTS (Hartmann, Hewett \& Calvet 1994; Muzerolle, Hartmann \& Calvet 1998; Muzerolle, Calvet \& Hartmann 1998, 2001), providing strong support to the magnetospheric model of accretion.

The average mass accretion rate for CTT disks in several known associations is $\sim 10^{-8} \mathrm{M}_{\odot} \mathrm{yr}^{-1}$ (Gullbring et al. 1998; Hartmann et al 1998.) For this low mass accretion rate, the infrared SED produced by the dust in the accretion disk is dominated by reprocessed stellar radiation intercepted by the disk curved surface (Kenyon \& Hartmann 1987; Calvet et al. 2000). Thus, why should one get into the trouble of including accretion in a model describing a disk? We discuss in this contribution why accretion plays a key role in disk structure and cannot be ignored.

Models of passive disks (i.e., disks heated only by stellar irradiation) have been proposed to explain the observed SEDs of CTTS and Herbig Ae stars from IR to mm wavelengths (Chiang \& Goldreich 1997). In contrast to power law models, where mass surface density and temperature of the disk are given by arbitrary power laws, the passive disk models treat in detail the energy balance between heating by absorbed stellar radiation and radiative cooling at each point. In recent years, powerful radiative transfer techniques have been developed for passive disk models, which result in successful accounting for several observed features (see Dullemond, van Zadelhoff \& Natta 2002; Dullemond 2002; Dullemond \& Natta 2003). However, even in the most sophisticated versions of these models, the disk mass surface density is still assumed to be an arbitrary power law of distance, $\Sigma=\Sigma_{0}\left(R / R_{0}\right)^{-p}$, and $\Sigma_{0}$ and $p$ are adjusted to fit a particular observation, with no link to the underlying physics of the disk.

On the other hand, in a steady accretion disk the mass accretion rate is related to the angular momentum flux produced by a viscous torque. As a consequence, the mass surface density is given by $\Sigma \propto \dot{M} / \nu_{t}$, where $\nu_{t}$ is the viscosity coefficient. It is common to adopt the $\alpha$ prescription (Shakura \& Sunyaev 1974), such that $\nu_{t}=\alpha c_{s} H$, where $c_{s}$ is the local sound speed, $H$ is the local gas scale height and $\alpha$ summarizes the unknown origin of the viscosity. Thus, instead of assuming an arbitrary power law for $\Sigma(R)$, the surface density is a result of the calculations in accretion disk models, given $\dot{M}$ (which can be independently quantified from observations), the temperature (which is calculated from viscous and irradiation heating) and $\alpha$ (which, in principle, can be estimated from magnetohydrodynamic simulations; however, in general, is taken to be a free constant parameter).

Irradiated accretion disk models show that even for $\dot{M} \sim 10^{-8} \mathrm{M}_{\odot} \mathrm{yr}^{-1}$, viscous dissipation is an important heating mechanism close to the mid-plane and close to the central star ( $R<1$ AU) (D'Alessio et al. 1997, 1999, 2001). On the other hand, stellar radiation intercepted by the disk upper layers is the most important heating mechanism of the disk atmosphere and the outer disk (Kenyon \& Hartmann 1987; Calvet et al. 1991, $1992=$ CMPD; D'Alessio et al. 1998, 1999, 2001). The upper layers of the atmosphere are hotter than the interior (CMPD), and this is a characteristic also present in the passive models mentioned above. These upper optically thin hot layers are probably responsible for some of the observed emission features (silicate $10 \mu \mathrm{m}$ band, Natta, Meyer \& Beckwith 2000; CO overtone emission, Najita et al. 1996). 
Their reprocessed radiation heats the disk interior, but do not contribute much to the continuum, specially when scattering of stellar radiation by atmospheric dust grains is included (D'Alessio et al. 1998; Dullemond \& Natta 2003).

In this paper we illustrate the importance of taken into account the accretion nature of the disk. First of all, the disk mass surface density is related to its mass accretion rate and viscosity, and the local viscous dissipation should be included as a heating source, even if the mass accretion rate is small. Second, the disk receives radiation from both the star and the accretion shocks at the stellar surface. This becomes particularly important for those objects where the accretion luminosity is comparable to the stellar luminosity, and has profound effects on the structure of their inner disk, as discussed below. It is specially relevant for high resolution interferometric observations, and opens up a number of possibilities to unveil the physics of accretion.

\section{Irradiated Rim}

Muzerolle et al. (2003a, MCHD) have presented near-infrared spectra of the excess continuum emission from the innermost regions of 9 CTTS. The shape of this excess is consistent with that of a single temperature black-body with $T \sim 1200-1400 \mathrm{~K}$, which are the expected sublimation temperatures for silicates under typical disk conditions (Pollack et al. 1994). The observed SEDs agree with that of a rim at the dust sublimation radius, receiving radiation frontally. A rim irradiated by the central star has been proposed by Natta et al. (2001), Dullemond, Dominik \& Natta (2001) to explain the observed excess at $3 \mu \mathrm{m}$ in the SEDs of Herbig Ae stars, and by Tuthill, Monnier \& Danchi (2001) and Monnier \& Millan-Gabet (2002) to explain interferometric near-IR images of disks around Herbig Ae and CTT stars. However, in the CTTS case, MCHD find no direct relationship between the radius of the rim and the stellar luminosity; rather, this radius increases with accretion luminosity. This is naturally explained when the fact that the disk is irradiated by both the stellar and the accretion luminosity is taking into account (MCHD.) In the following section we outline the model for the temperature at the rim atmosphere, which can explain the MCHD observations, and we show how the rim radius depends on the accretion and stellar luminosities as well as on the dust properties.

\subsection{Model for the irradiated Rim of an accretion disk}

We follow a simplified version of CMPD's treatment to describe the radial temperature distribution in the rim atmosphere, assuming it has plane-parallel geometry and constant opacity. The radiation field is separated into two frequency ranges, one characteristic of the disk (and the related quantities have subindex "d") and one characteristic of the incident radiation (with subindex "i"). In the case of the rim, the incident field is given by the superposition of the stellar radiation field (characterized by the star effective temperature, $T_{*}$ ) and the accretion shock radiation field (characterized by a temperature $\sim 8000 \mathrm{~K}$ and a luminosity $L_{\text {shock}}$, CG98).

For simplicity, we assume that the scattering of incident radiation is essentially forward, which is equivalent to take the albedo to be equal zero in CMPD equations (i.e., $J_{s}=H_{s}=0$ ). Also, we assume that the incident radia- 
tion reaches the rim with an angle 0 relative to the normal to the surface, i.e., $\cos \theta_{0}=\mu_{0}=1$, and that there is no other heating source than the incident radiation. Thus, at every depth into the rim atmosphere the net outward radial flux at the disk frequency range is equal to the incident stellar flux, i.e,

$$
H_{d}\left(\tau_{s}\right)=\frac{F_{0}}{4 \pi} e^{-\tau_{s}}
$$

where $F_{0}=\left(L_{*}+L_{\text {shock }}\right) / 4 \pi R^{2}$ and $\tau_{s}$ is the optical depth to the stellar radiation in the incident direction (i.e., along rays parallel to the disk mid-plane, perpendicular to a cylindrical surface at the dust sublimation radius). 1978),

The zeroth moment of the transfer equation can be written as (Mihalas

$$
\frac{d H_{d}}{d \tau_{d}}=J_{d}-S_{d}
$$

where $\tau_{d}$ is the optical depth at the disk frequency range, $S_{d}$ is the local source function and $J_{d}$ is the mean intensity. CMPD assume strict LTE, i.e., $S_{d}=$ $B_{d}=\sigma T_{d}^{4} / \pi$. Here, we will include a term corresponding to the emissivity by scattering at the disk frequency range, i.e., $S_{d}=\left(\kappa_{d} B_{d}+\sigma_{d} J_{d}\right) /\left(\kappa_{d}+\sigma_{d}\right)$, assuming isotropic scattering at this frequency range (Mihalas 1978). With this source function, the zeroth moment of the transfer equation is given by

$$
B_{d}=J_{d}-\frac{\chi_{d}}{\kappa_{d}} \frac{d H_{d}}{d \tau_{d}} .
$$
tion is

Using the Eddington approximation, the first moment of the transfer equa-

$$
\frac{d J_{d}}{d \tau_{d}}=-3 H_{d}=-3 \frac{F_{0}}{4 \pi} e^{-q \tau_{d}}
$$

where we have defined $q=\tau_{s} / \tau_{d}$. Integrating $J_{d}$ from eq(4), using $J_{d}(0)=$ $2 H_{d}(0)$ as the boundary condition, and substituting $J_{d}$ and $d H_{d} / d \tau_{d}$ from eq(1) in eq(3), the temperature as a function of $\tau_{d}$ can be written as

$$
T_{d}\left(\tau_{d}\right)^{4}=\frac{F_{0}}{4 \sigma_{R}}\left[\left(2+\frac{3}{q}\right)+\left(q \frac{\chi_{d}}{\kappa_{d}}-\frac{3}{q}\right) e^{-q \tau_{d}}\right] .
$$

The temperature at the innermost radius of the rim, where $\tau_{d}=\tau_{s}=0$, should be equal to the silicates sublimation temperature (assuming the inner gaseous disk has a negligible radial optical depth). Thus, the rim radius $R_{s u b}$ is

$$
R_{s u b}=\left[\left(\frac{L_{*}+L_{\text {shock }}}{16 \pi \sigma_{R}}\right)\left(2+q \frac{\chi_{d}}{\kappa_{d}}\right)\right]^{1 / 2} \frac{1}{T_{s u b}^{2}} .
$$

$\mathrm{Eq}(6)$ is essentially similar to the equation for the rim radius proposed by Tuthill et al. (2001), except for the enhanced heating due to the accretion shocks at the stellar surface (given by $L_{\text {shock }}$ ), and the addition of 2 to the opacity term inside the parenthesis, which arises from the explicit inclusion of the disk radiation field.

The temperature at the level where $\tau_{d}=2 / 3$, which characterizes the emergent flux, is 


$$
T^{4}\left(\tau_{d}=\frac{2}{3}\right)=T_{e f f}^{4}=\left(\frac{L_{*}+L_{s h o c k}}{16 \pi \sigma_{R}}\right)\left(2+\frac{3}{q}+\left(\frac{q \chi_{d}}{\kappa_{d}}-\frac{3}{q}\right) e^{-2 q / 3}\right] .
$$

Modeling of the observed near IR SEDs of CTTS indicates that the contribution of the optically thin layers of the rim (with dust) to the SED is negligible, so that the emergent intensity $I_{\nu}$ differs from $B_{\nu}\left(T_{\text {eff }}\right)$ by only few percent (MCDH). Also, we find that $T_{\text {eff }}$ is lower than $T(0)=T_{\text {sub }}$ within $\sim 100 \mathrm{~K}$, so that the rim atmosphere is almost radially isothermal (MCDH).

Finally, the emergent flux is $F_{\nu}=I_{\nu} \Omega(i)$, where $\Omega(i)$ is the solid angle subtended by the rim as seen by the observer and $i$ is the inclination angle between the disk symmetry axis and the line of sight. The rim height is $z_{\text {rim }}$, defined as the height where the optical depth to the stellar radiation impinging radially becomes unity at the rim radius $R_{s u b}$. For an accretion disk this height can be estimated considering the rim to be vertically isothermal with a mass surface density given by $\Sigma=\dot{M} / 6 \pi \nu_{t}\left[1-\left(R_{*} / R_{\text {sub }}\right)^{1 / 2}\right]$ (Muzerolle et al. 2003b). We find typically $z_{\text {rim }} \sim 4 H_{\text {rim }}$, where $H_{\text {rim }}$ is the gas vertical scale height of the rim (assumed to be vertically isothermal).

Defining $\delta=z_{\text {rim }} \tan i / R_{\text {sub }}$, the solid angle of the rim is

$$
\begin{gathered}
\Omega(i)=\pi\left(\frac{R_{s u b}}{d}\right)^{2} \cos i \quad \text { for } \delta>1 \\
\Omega(i)=4\left(\frac{R_{s u b}}{d}\right)^{2} \frac{\cos i}{2}\left(\sin ^{-1} \delta+\delta \sqrt{1-\delta^{2}}\right) \quad \text { for } \delta<1
\end{gathered}
$$

Both expressions multiplied by $B_{\nu}\left(T_{\text {eff }}\right)$, are consistent with the formulae for the emergent flux given in the Appendix B of Dullemond et al. (2001), except for our different definition of $R_{s u b}$, which assumes that the dust is optically thin at the sublimation radius, and for using a $T_{\text {eff }}$ different from the sublimation temperature.

\section{Results}

In this section, we discuss irradiated accretion disk models, including the rim, that explain high resolution IR observations or SEDs of different objects. The rim model just described fits the near IR spectra of several CTTS, with rim radii in the range 0.07-0.54 $\mathrm{AU}$, increasing with higher stellar and accretion luminosities (MCDH). Here, we first show that this model can explain the recent interferometric observations presented by the Keck team. Then, we present models for two systems with widely different accretion luminosities, to illustrate the effects of accretion.

\subsection{DG Tau}

Keck interferometric observations of DG Tau (Colavita et al. 2003) showed that the system was resolved at $\mathrm{K}$, and derived a size of $\sim 0.12-0.24 \mathrm{AU}$. This radius is $\sim 3$ times larger than the dust sublimation radius inferred assuming the rim is irradiated only by the central star. Specifically, for an optically thick 
wall, $R_{s u b}^{\prime}=\left(L_{*} / 16 \pi \sigma_{R} T_{s u b}^{4}\right)^{1 / 2}$. Using typical parameters for a K7-M0 central star $\left(T_{*}=4000 \mathrm{~K}, R_{*}=2 \mathrm{R}_{\odot}\right.$ and $\left.M_{*}=0.3 \mathrm{M}_{\odot}\right)$, and a silicate sublimation temperature $T_{s u b}=1400 \mathrm{~K}, R_{\text {sub }}^{\prime} \sim 0.07$ AU. However, DG Tau is a "continuum star", with a mass accretion rate $\dot{M}=5 \times 10^{-7} \mathrm{M}_{\odot} \mathrm{yr}^{-1}$ (Gullbring et al. 2000). Assuming the typical dust properties derived for the sample of CTTS studied by MCHD (i.e., $q=1, \chi_{d} / \kappa_{d}=2$ ), we find that $R_{s u b} \sim 0.2 \mathrm{AU}$, which is $\sim 3$ time larger than $R_{s u b}^{\prime}$, and consistent with the observed inner radius of DG Tau.

\subsection{LkCa 15}

We have calculated a model for the structure and emission of $\mathrm{LkCa} \mathrm{15,} \mathrm{which} \mathrm{is}$ a low mass accretion rate CTTS. The details of the calculation of an irradiated accretion disk model are described by D'Alessio et al. (1998, 1999, 2001). The input parameters constrained by observations are $\dot{M}=1.3 \times 10^{-9} \mathrm{M}_{\odot} \mathrm{yr}^{-1}$, $T_{*}=4350 \mathrm{~K}, R_{*}=1.5 \mathrm{R}_{\odot}, M_{*}=0.8 \mathrm{M}_{\odot}$ (Hartmann et al. 1998) Figure 1right panel shows the comparison between the observed and predicted SEDs. We explain the shape of the observed SED between $60 \mu \mathrm{m}$ to $1.3 \mathrm{~mm}$, assuming that the outer disk $(R>3 \mathrm{AU})$ has a uniform grain size distribution characterized by $n(a) \propto a^{-3.5}$, with $a_{\max }=1 \mathrm{~mm}$. These are typical dust properties such that an irradiated disk model with the median $\dot{M}$ and a typical central star, fits the median SED of CTTS in Taurus ( D'Alessio et al.' 2001). We adopt a maximum disk radius $R_{d}=200 \mathrm{AU}$ and $\alpha=0.001$, corresponding to a disk mass $\sim 0.04 \mathrm{M}_{\odot}$. Qi et al. (2003) find that the $\mathrm{mm}$ SED does not show a single spectral index, which is naturally explained, by taking into account the contribution of optically thick regions at small radii.

In this model, the disk rim is placed at $R_{\text {sub }} \approx 0.06 \mathrm{AU}$. Being $L_{*}>L_{\text {shock}}$, the rim is mostly heated by stellar radiation. There is a shadowed region between the rim and $\sim 0.16 \mathrm{AU}$, which produces a negligible contribution to the SED. We assume that this region is only being heated by viscous dissipation. Finally, there is an intermediate region between $\sim 0.16$ and $3 \mathrm{AU}$, where the disk atmosphere is characterized by smaller grains than the disk interior and the disk outer regions. This region is responsible for the observed $10 \mu \mathrm{m}$ silicate band which is formed in the upper layer, heated by direct stellar radiation (Natta et al. 2000). In order to fit the shape of the silicate band, we have used optical constants for olivine, $\mathrm{MgFe} \mathrm{SiO}_{4}$ (Jäger et al. 1998). To produce a continuum around 10 $\mu m$ consistent with the observed value, the dust to gas mass ratio of the upper atmosphere should be a factor $\sim 0.05$ smaller that the standard value, probably reflecting that dust growth and settling has taken place in the inner disk. In principle, the details of this model can be tested by high resolution observations capable of resolving scales between 0.4 and 20 mas.

\subsection{DR Tau}

DR Tau is a continuum star, characterized by a strong UV/optical continuum. We have calculated a model for the disk of DR Tau, with the following parameters $(\mathrm{MCHD}) T_{*}=4000 \mathrm{~K}, R_{*}=1.9 \mathrm{R}_{\odot}$ and $M_{*}=0.9 \mathrm{M}_{\odot}$ for the central star. We take $\dot{M}=5 \times 10^{-7} \mathrm{M}_{\odot} \mathrm{yr}^{-1}$, roughly consistent with the value inferred from the optical/UV excess (Kenyon et al. 1994; Gullbring et al. 2000). A uniform 


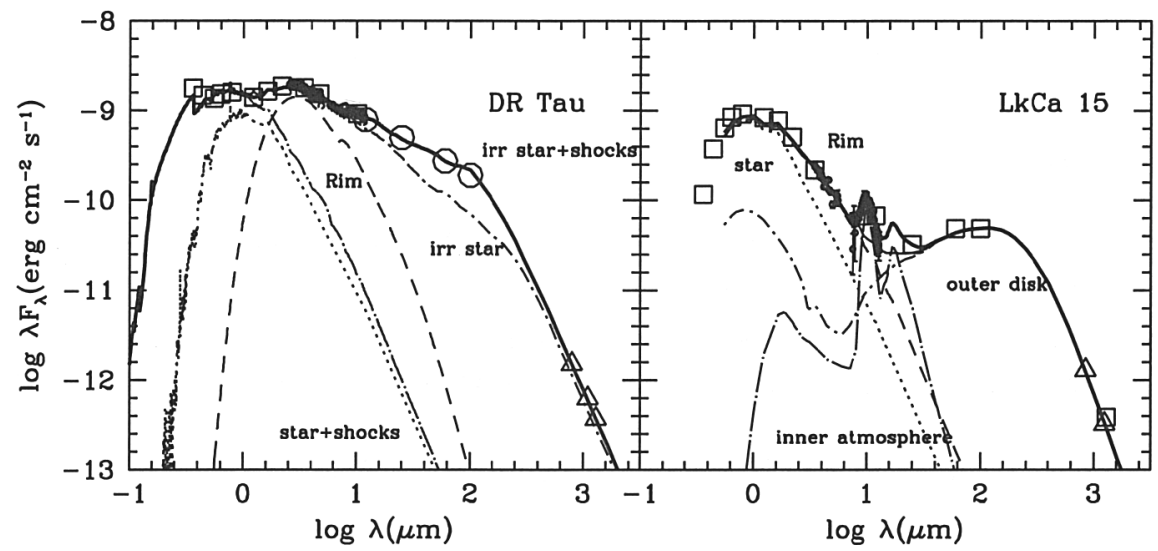

Figure 1. Right panel:SED of LkCa15 showing different contributions. Left panel: SED of DR Tau showing the contributions of different zones. For comparison, also a SED of the same disk model only irradiated by the central star is shown. See D'Alessio et al. (2003) for references to observational data.

maximum grain size $a_{\max }=1 \mathrm{~mm}, \alpha=0.01, R_{d}=50 \mathrm{AU}$, corresponding to a disk mass $M_{d} \sim 0.2 \mathrm{M}_{\odot}$. In contrast to the case of LkCa 15 , accretion luminosity determines the structure and emission of the entire disk for this object. The rim radius is $R_{s u b} \sim 0.15 \mathrm{AU}$, and it is heated mostly by the hot continuum produced in the accretion shocks at the stellar surface. Figure 1-left panel shows that the rim emission dominates the SED between 3 and $6 \mu \mathrm{m}$. Local viscous dissipation is important in heating the disk photosphere for $R \lesssim 3 \mathrm{AU}$ and heating the mid-plane at every radius $(R \lesssim 50 \mathrm{AU})$. However, the photosphere of the outer disk is heated mostly by radiation from the accretion shocks. This model can also be tested by high resolution observations.

\section{Conclusions}

We have shown that accretion plays an important role in determining the structure and emission of disks around CTTS and cannot be dismissed. The surface density of the disk depends on the mass accretion rate, and moreover, accretion energy determines the location of the transition between dust and gas in the inner disks, with direct implications for interferometric observations. In particular, by including the contribution of accretion, the puzzling Keck interferometer observations of DG Tau, with an disk inner radius larger than the sublimation radius expected from stellar luminosity alone, can be understood.

Acknowledgments. PD would like to thank the organizing Comittee of IAU Symposium 221 for a travel grant. MS acknowledges a grant from NASA 
Origins of Solar Systems NAG5-9475. Support for this research comes from NASA Origins of Solar Systems grant NAG 5-9670 and grants from UNAMDGAPA/PAPIIT and Conacyt, México.

\section{References}

Calvet, N., Hartmann, L., \& Strom, S. E. 2000, Protostars and Planets IV, 377 Calvet, N. \& Gullbring, E. 1998, ApJ, 509, 802

Calvet, N., et al. 1992, Rev. Mexicana Astron. Astrofis., 24, 27

Calvet, N., Patiño, A., Magris, G. C., \& D'Alessio, P. 1991, ApJ, 380, 617 (CPMD)

D'Alessio, P., Calvet, N., \& Hartmann, L. 2001, ApJ, 553, 321

D'Alessio, P. et al. 1999, ApJ, 527, 893

D'Alessio, P., Cantó, J., Calvet, N., \& Lizano, S. 1998, ApJ, 500, 411

Dullemond, C. P. \& Natta, A. 2003, A\&A, 408, 161

Dullemond, C. P. 2002, A\&A, 395, 853

Dullemond, C. P., van Zadelhoff, G. J., \& Natta, A. 2002, A\&A, 389, 464

Dullemond, C. P., Dominik, C., \& Natta, A. 2001, ApJ, 560, 957

Gullbring, E., Hartmann, L., Briceño, C., \& Calvet, N. 1998, ApJ, 492, 323

Hartmann, L., Kenyon, S. J., \& Calvet, N. 1993, ApJ, 407, 219

Jaeger, C. et al. 1998, A\&A, 339, 904

Kenyon, S. J., \& Hartmann, L. 1987, 323, 174

Kenyon, S. J., \& Hartmann, L. 1995, ApJS, 101, 117 (KH95)

Knigl, A. 1991, ApJ, 370, L39

Mihalas, D. 1978, Stellar Atmospheres (San Francisco: Freeman)

Millan-Gabet, R., Schloerb, F. P., Traub, W. A., Malbet, F., Berger, J. P., \& Bregman, J. D. 1999, ApJL, 513, L131

Muzerolle, J.,Calvet, N.,Hartmann, L. \& D'Alessio, P. 2003a, ApJL, submitted (MCHD)

Muzerolle, J., D'Alessio, P., Calvet, N., \& Hartmann, L. 2003b, ApJ, submitted Muzerolle, J., Hartmann, L., \& Calvet, N. 1998, AJ, 116, 2965

Muzerolle, J., Calvet, N., \& Hartmann, L. 2001, ApJ, 550, 944

Najita, J. et al. 1996, ApJ, 462, 919

Natta, A., Meyer, M. R., \& Beckwith, S. V. W. 2000, ApJ, 534, 838

Qi, C., Kessler, J., Koerner, D., Sargent, A., \& Blake, G. 2003, submitted to ApJ

Pollack, J. B., Hollenbach, D., Beckwith, S., Simonelli, D. P., Roush, T., \& Fong, W. 1994, ApJ, 421, 615 (P94)

Shakura, N. I., \& Sunyaev, R. A. 1973, A\&A, 24, 337

Shu, F. H. et al. 1994, ApJ, 429, 781

Monnier, J. D. \& Millan-Gabet, R. 2002, ApJ, 579, 694

Tuthill, P. G., Monnier, J. D., \& Danchi, W. C. 2001, Nature, 409, 1012 